International Journal of Biology, Pharmacy and Allied Seiences (IJBPAS)

'A Bridge Betuen Caboratory and QRendo'

WwW.ibpas.com

\title{
ON-ALCOHOLIC FATTY LIVER DISEASE WITH METABOLIC SYNDROME
}

\author{
ARAVIND C ${ }^{1}$ AND APARNNA $\mathbf{S}^{\mathbf{2}^{*}}$ \\ 1, 2: Department of General Medicine, Sri Lakshmi Narayana Institute of Medical Sciences, \\ Pondicherry, India \\ *Corresponding Author: Dr. Aparnna Sudhakar: E Mail: aparnna13@gmail.com
} Received 17 $7^{\text {th }}$ Oct. 2020; Revised $16^{\text {th }}$ Nov. 2020; Accepted 12 ${ }^{\text {th }}$ Dec. 2020; Available online $1^{\text {st }}$ Jan. 2021 https://doi.org/10.31032/IJBPAS/2021/10.1.1009

\begin{abstract}
Non alcoholic fatty liver disease is the most common health burden in developed countries. The spectrum of liver damage includes NASH, advanced fibrosis and rarely progresses to cirrhosis. Insulin resistance, oxidative stress, lipid peroxidation, mitochondrial dysfunction play a major role in the development of NAFLD. Our study strongly suggest that the association of non alcoholic fatty liver disease as a possible component in metobolic syndrome. This cross sectional study was conducted in Vazhudavur, Pondicherry from Nov 2018- Jan 2019. 1000 people participated in this study whose age was 30 and above. Anthropometry measurements, random blood sugar, blood pressure measurements, BMI, and USG were evaluated to screen for metabolic syndrome. A questionnaire was used to obtain information regarding history and quantity of alcohol consumption. Metabolic syndrome was defined in all participants based on national cholesterol education program adult treatment panel 111 (2001) (NCEP/ATP 111) for clinical diagnosis of metabolic syndrome. Non alcoholic fatty liver disease was detected in $33.1 \%$ and $27.4 \%$ of men and women respectively. NAFLD is mainly associated with the development of MetS. However, it was not possible to determine whether NAFLD preceded the development of MetS.
\end{abstract}

\footnotetext{
Keywords: Non-alcoholic fatty liver disease, Metabolic syndrome, NCEP/ATP-III, Waist circumference, Hyperglycemia
} 


\section{INTRODUCTION}

Non-alcoholic fatty liver disease (NAFLD) is a spectrum of disease ranging from simple steatosis to steatohepatitis, with degrees of inflammation and fibrosis, which progresses to cirrhosis and hepatocellular carcinoma [1]. NAFLD is now more common than alcoholic liver disease owing to the rapid rise in the prevalence of obesity, and NAFLD is the most common cause of abnormal liver function tests [2]. Previous studies shown that around $80 \%$ of population with elevated liver enzymes have NAFLD. 30\% of the general adult population and up to $60-70 \%$ of obese patients with diabetes mellitus patients have NAFLD.

MetS is a cluster of conditions with increased blood pressure, high blood sugar, excess body fat around the waist, abnormal triglyceride levels-that occur together, increasing the risk of developing heart disease, stroke, and Diabetes. NAFLD and MetS have been linked by relationships between central obesity, steatosis, and insulin resistance [3].

\section{MATERIALS AND METHODS}

\section{Study Population}

This cross sectional study was conducted from Nov 2018 to Jan 2019. The lifestyle nature of this town is rural and in this study, the demographic and anthropometric characteristics of the participants were documented in the questionnaire. The questioners were trained how to fill the questionnaire and how to measure systolic and diastolic blood pressure, waist circumference, hip circumference, weight and height. The blood samples were transferred to our lab within an hour under standard conditions. We included $\geq 30$ year-old volunteers who had undergone ultrasonography confirming fatty liver. We excluded those who were alcohol users, who were being treated for NAFLD or using medications such as amiodarone, tamoxifen, pregnant women and $\mathrm{HbsAg}$ positive patients.

\section{Measurements}

The demonstration of areas of hyperechogenicity on sonography is used in the diagnosis of NAFLD in all individuals. Sonograms were obtained using static and real time scanning with commercially obtained units. A transducer with $3.5-\mathrm{MHz}$ was routinely used, with a transducer of 2.25-MHz reserved for areas to penetrate is difficult. The patients are examined in both supine and left posterior oblique positions, with longitudinal, transverse, and oblique scanning planes.

The severity of echogenicity were graded as $0,1,2 \& 3$

Systolic and diastolic blood pressures were measured as duplicate on 
the left upper arm and the average used for analysis.

WC between the lowest rib and the iliac crest at the level of umbilicus was measured in duplicate to the $\mathrm{cm}$ using a flexible tape.

Plasma glucose for the subjects was measured using the glucose-peroxidase colorimetric enzymatic method with a sensitivity of $5 \mathrm{mg} / \mathrm{dL}$ and intra-assay coefficients of variation (CV) of $1.7 \%$ in the lower limit and $1.4 \%$ in the upper limit concentrations. Inter-assay coefficients of variation $(\mathrm{CV})$ for the assay were $1.1 \%$ in the lower limit and $0.6 \%$ in the upper limit concentrations respectively.
Serum cholesterol and triglyceride for all individuals were measured after $12-$ 14 hours of fasting with colorimetric method with a sensitivity of $5 \mathrm{mg} / \mathrm{dl}$. Intraassay and inter-assay CV for the assay were $1.6 \%$ and $1.1 \%$ in lower limit and $0.6 \%$ and $0.9 \%$ for upper limit concentrations. HDL$\mathrm{C}$ were measured after precipitation of the apolipoprotein B containing lipoproteins with phosphotungstic acid.

\section{Metabolic Syndrome Criteria}

Two different criteria were used in this study, NCEP/ATP-III and CCDMIA. MetS was defined as the presence of three or more of the presented parameters in below Table 1.

Table 1: Two Criteria for Clinical Diagnosis of Metabolic Syndrome which was used in this Study

\begin{tabular}{|c|c|c|}
\hline & \multicolumn{2}{|c|}{ Criteria } \\
\hline Variables & NCEP/ATP-III & CCDMIA \\
\hline Abdominal obesity & WC $>102 \mathrm{~cm}$ in men and $>88 \mathrm{~cm}$ in women & WC $\geq 95$ in both sexes \\
\hline Hypertriglyceridemia & TG $\geq 150 \mathrm{mg} / \mathrm{dL}$ & TG $\geq 150 \mathrm{mg} / \mathrm{dL}$ \\
\hline Low HDL-C level & $<40 \mathrm{mg} / \mathrm{dL}$ in $\mathbf{m e n}$ and $<50 \mathrm{mg} / \mathrm{dL}$ in women & $<40 \mathrm{mg} / \mathrm{dL}$ in $\mathrm{men}$ and $<50 \mathrm{mg} / \mathrm{dL}$ in women \\
\hline Hypertension & SBP/DBP $\geq 130 / 85 \mathrm{mmHg}$ & SBP/DBP $\geq 130 / 85 \mathrm{mmHg}$ \\
\hline Hyperglycemia & FBS $\geq 110 \mathrm{mg} / \mathrm{dL}$ & FBS $\geq 100 \mathrm{mg} / \mathrm{dL}$ \\
\hline
\end{tabular}

NCEP/ATP-III, National Cholesterol Education Program Adult Treatment Panel III; CCDMIA, Criteria for Clinical Diagnosis of metabolic syndrome in Iranian Adults; WC, Waist Circumference; TG, Triglyceride, SBP, Systolic Blood Pressure;

\section{RESULTS} DBP, Diastolic Blood Pressure; FBS, Fasting Blood Sugar.

We enrolled $359(33.3 \%)$ men and $647 \quad(68.7 \%)$ women. Among the individuals $\geq 30$ years there were 202 $(80.8 \%)$ men and $598(90.2 \%)$ women with a male to female ratio of $1: 2.45$. below table shows the frequency, percentage and mean \pm SD age of all participants, those $\geq 30$ years, and those $\geq 30$ years that had NAFLD with different grades. The men to women ratio in all participants, those $\geq 30$ years, and those $\geq 30$ years who had NAFLD were 1:2.19, 1:2.45, and 1:2.03, respectively. Mild NAFLD was more prevalent in both men and women. However, moderate and severe NAFLD was more prevalent in women compared with men $(p>0.05)$. Among those $\geq 18$ years, men were about 4 years older than women $(p<0.001)$. NAFLD was detected in $33.1 \%$ and $27.4 \%$ of men and women, respectively (Table 2). 
The prevalence of MetS and its related NAFLD grades according to NCEP/ATPIII and CCDMIA are presented. As demonstrated, the number of men with MetS based on NCEP/ATPIII was higher than CCDMIA. In the opposite trend, the number of women with MetS based on CCDMIA was higher than NCEP/ATPII. Among men, according to the NCEP/ATPIII and CCDMIA criteria, $65.9 \%$ and $30.1 \%$ had MetS, respectively. These percentages in women were $64.6 \%$ and $73.7 \%$, based on NCEP/ATPIII and CCDMIA criteria, respectively. Also, most of the patients with MetS had mild NAFLD in both sexes based on both criteria
NCEP/ATP-III, National Cholesterol Education Program Adult Treatment Panel III; CCDMIA, Criteria for Clinical

\section{Diagnosis of Mets in Adults}

The frequency and its percentage, 95\% CI, and odds ratio for each component of MetS in patients with NAFLD is presented. It shows that there were no significant differences between the two genders in none of the components $(p>0.05)$. Although, OR for hyperglycemia and abdominal obesity were approximately high in CCDMIA criteria (0.9613 and 1.2082, respectively), the respected differences were not statistically significant.

Table 2: Frequency, Percentage and mean \pm SD of the age of the Participants Based on Different Categories

\begin{tabular}{|c|c|c|c|c|c|}
\hline Variables & Grade a & Men & Women & & \\
\hline & & Frequency (\%) & Age (years) & Frequency (\%) & Age (years) \\
\hline$\geq 30$ years old & & $202(29)$ & $44.1 \pm 16.4 *$ & $598(71)$ & $40.2 \pm 30.7$ \\
\hline \multirow{3}{*}{ NAFLD } & All & $66(32.9)$ & $46.5 \pm 13.4$ & $163(27.4)$ & $46.5 \pm 12.0$ \\
\cline { 2 - 6 } & I & $42(63.2)$ & $45.5 \pm 13.6$ & $100(61.3)$ & $44.6 \pm 11.2$ \\
\cline { 2 - 6 } & II & $20(30.9)$ & $47.9 \pm 12.2$ & $52(31.8)$ & $49.2 \pm 12.3$ \\
\cline { 2 - 6 } & III & $4(5.9)$ & $50.4 \pm 15.9$ & $11(6.9)$ & $\mathbf{5 0 . 2} \pm 13.9$ \\
\hline
\end{tabular}

\section{DISCUSSION}

This study assessed the prevalence of MetS and its associated components in different grades of NAFLD. We found no difference between both men and women in NAFLD prevalence nor in MetS based on both criteria. The diagnosis of NAFLD requires a invasive and non-invasive tests. Mild to moderately increased levels of aspartate aminotransferase and alanine aminotransferase were the most common findings [8]. However some studies suggest that the use of liver enzymes as a marker of NAFLD underestimate its prevalence [9]. Ultrasound has a sensitivity of $84 \%$ and $76 \%$ and a specificity of $90 \%$ and $84 \%$ in detecting steatosis and increased fibrosis, respectively [8], and therefore we used this noninvasive easy technique in our study.

The prevalence of NAFLD was determined to be $27.4 \%$ in this study, which is different from the one report from Taiwan [10]. Other reports show rates of 10-30\% from Taiwan, Japan, India [11], 
and USA, Europe, and Indonesia [12, 13]. Also, the prevalence of MetS in patients with NAFLD ranged from $61-65 \%$, based on CCDMIA and NCEP/ATPIII, respectively which was 1.8 to 3.1 times more than previous reports. This is moderately higher than that reported by Uchil et al. among Indian adult patients with NAFLD (47\%) [8]. The higher prevalence of NAFLD in men compared with women in our study $(33.1 \%$ vs. $27.4 \%$ ) was similar to a previous report from Taiwan [8]. Despite the difference in criteria for the two components of MetS and gender, the prevalence of mild NAFLD (grade I) in patients with MetS was approximately high (about 50\%). In the present study, hyperglycemia (OR: 1.21, 95\% CI: 0.90-1.62) and abdominal obesity (OR: 0.96, 95\% CI: 0.72-1.28), based on CCDMIA were the most NAFLD related components of MetS. NAFLD and MetS have been tangentially linked in the association with central obesity and insulin resistance [3]. This fact was highlighted in a large multicenter study, which showed that metabolic syndrome and insulin resistance were associated with NAFLD by multivariate analysis [15]. In the adult population of the Tehran Lipid and Glucose Study (TLGS), MetS was found in $42 \%$ of women and $24 \%$ of men with a total agestandardized prevalence of $33.7 \%[\mathbf{1 4}, \mathbf{1 5}]$.
Also, it has been reported that obesity is now recognized as a major health problem and is the prominent underlying factor in MetS [16]. The findings from our study support the relationship between obesity and hyperglycemia with MetS. Marchesani et al. showed that $80 \%$ of patients with NAFLD were obese [17] that is obviously higher than our estimation $(47.2 \%$ in NCEP/ATPIII and 52.8\% in CCDMIA).

The prevalence of MetS differed widely in different studies according to the population sample studied and the diagnostic criteria used. In our study population, the prevalence of MetS $(65.25 \%$ and $51.90 \%)$ was different based on the diagnostic criteria used (NCEP/ATP-III or CCDMIA). The prevalence of MetS tended to be higher in men when the NCEP/ATP-III criteria were used, and tended to be higher in women when the CCDMIA were used. For these two definitions, the greatest difference is of the diagnosis of hyperglycemia and abdominal obesity.

Our study suffered from three important limitations: First, in our participants, volunteers women were more refereed than men due to occupational busy of men; Second, NAFLD diagnosis and grading was made only based on sonography. The most important reasons for this limitation were performing 
sonography in asymptomatic volunteer population and also its inexpensiveness and non-invasiveness; Third, our participants did not have any control group.

In conclusion, although our study demonstrated that NAFLD was associated with MetS, from this analysis it was not possible to determine whether NAFLD predating the development of MetS. Moreover, difference in detected MetS prevalence between NCEP/ATP-III and CCDMIA demonstrated that criteria in MetS are still a controversial subject and definition of better and more effective criteria for diagnosis of MetS in different population is seriously needed. Further well-designed case-control studies with follow-up and using of concurrent different criteria are needed to elucidate the causative relationship between these two conditions.

\section{REFERENCES}

[1] Pacifico L, Nobili V, Anania C, Verdecchia P, Chiesa C. Pediatric nonalcoholic fatty liver disease, metabolic syndrome and cardiovascular risk. World J Gastroenterol. 2011; 17: 3082-91. [PMC free article] [PubMed].

[2] Sattar N, Forrest E, Preiss D. Nonalcoholic fatty liver disease. Brit Med J. 2014; 349: g4596.

doi: 10.1136/bmj.g4596. [PMC free article] [PubMed] [CrossRef].
[3] Streba LAM, Carstea D, Mitruţ P, Vere CC, Dragomir N, Streba CT. Nonalcoholic fatty liver disease and metabolic syndrome: a concise review. Rom J Morphol Embryol. 2008; 49: 13-20. [PubMed].

[4] Quinn SF, Gosink BB. Characteristic sonographic signs of hepatic fatty infiltration. Am J Roentgenol. 1985; 145: 753-5. [PubMed].

[5] Saadeh S, Younossi ZM, Remer EM, Gramlich T, Ong JP, Hurley M. et al. The utility of radiological imaging in nonalcoholic fatty liver disease. Gastroenterology. 2002; 123: 745-50. doi: 10.1053/gast.2002.35354. [PubMed] [CrossRef].

[6] National Heart Lung and Blood Institute. Third report of the National Cholesterol Education Program (NCEP) expert panel on detection, evaluation, and treatment of high blood cholesterol in adults (Adult Treatment Panel III): final report. Circulation. 2002; 106: 3143-421. doi: $\quad$ 10.1001/jama.285.19.2486. [PubMed] [CrossRef].

[7] Azizi F, Hadaegh F, Khalili D, Esteghamati AR, Hosseinpanah F, Delavari AR. et al. Appropriate definition of metabolic syndrome among Iranian adults: Report of the Iranian National Committee of 
Obesity. Arch Iran Med. 2010; 13: 426-8. [PubMed].

[8] Uchil D, Pipalia D, Chawla M, Patel R, Maniar S, Narayani Narayani. et al. Non-alcoholic fatty liver disease (NAFLD)-the hepatic component of metabolic syndrome. J Assoc Physicians India. 2009; 57: 201-4. [PubMed].

[9] Chalasani N, Younossi Z, Lavine JE, Diehl AM, Brunt EM, Cusi K. et al. The diagnosis and management of non-alcoholic fatty liver disease: Practice Guideline by the American Association for the Study of Liver Diseases, American College of Gastroenterology, and the American Gastroenterological Association. Hepatology. 2012; 55: 2005-23. doi: 10.1002/hep.25762. [PubMed] [CrossRef].

[10]Chen SH, He F, Zhou HL, Wu HR, Xia C, Li YM. Relationship between nonalcoholic fatty liver disease and metabolic syndrome. J Digest Dis. 2011; 12: 125-30. doi:10.1111/j.1751-2980.2011.00487.x. [PubMed] [CrossRef].

[11]Cho LW. Metabolic syndrome. Singapore Med J. 2011; 52: 779-85. [PubMed]

[12] Tsai C-H, Li T-C, Lin C-C. Metabolic syndrome as a risk factor for nonalcoholic fatty liver disease.
South Med J. 2008; 101: 900-5. doi: 10.1097/SMJ.0b013e31817e8af9.

[PubMed] [CrossRef].

[13]Hadaegh F, Zabetian A, Harati H, Azizi F. Metabolic syndrome in normal-weight Iranian adults. Ann Saudi Med. 2007; 27: 18-24. [PMC free article] [PubMed].

[14]Azizi F, Salehi P, Etemadi A, ZahediAsl S. Prevalence of metabolic syndrome in an urban population: Tehran Lipid and Glucose Study. Diabetes Res Clin Pract. 2003; 61: 29-37.

doi: $\quad$ 10.1016/S0168-8227(03)00066-4. [PubMed] [CrossRef].

[15]Caballería L, Pera G, Auladell MA, Torán P, Muñoz L, Miranda D. et al. Prevalence and factors associated with the presence of nonalcoholic fatty liver disease in an adult population in Spain. Eur J Gastroenterol Hepatol. 2010; 22: 2432.

doi:10.1097/MEG.0b013e32832fcdf0. [PubMed] [CrossRef].

[16]Chen C-H, Huang M-H, Yang J-C, Nien C-K, Yang C-C, Yeh Y-H. et al. Prevalence and risk factors of nonalcoholic fatty liver disease in an adult population of Taiwan: metabolic significance of nonalcoholic fatty liver disease in nonobese adults. $\mathrm{J}$ 
Clin Gastroenterol. 2006; 40: 745-52.

[PubMed].

[17]Marchesini G, Brizi M, Bianchi G, Tomassetti S, Bugianesi E, Lenzi M. et al. Nonalcoholic fatty liver disease a feature of the metabolic syndrome.

Diabetes. 2001; 50: 1844-50. doi:

10.2337/diabetes.50.8.1844.

[PubMed] [CrossRef]. 